Review Article

\title{
Prevalence of Malaria among Adults in Ethiopia: A Systematic Review and Meta-Analysis
}

\author{
Fasil Adugna Kendie $(\mathbb{D}$, Tamirat Hailegebriel W/kiros $(\mathbb{D}$, Endalkachew Nibret Semegn $(\mathbb{D}$, \\ and Melaku Wale Ferede
}

Department of Biology, Bahir Dar University, P.O. Box 79, Bahir Dar, Ethiopia

Correspondence should be addressed to Fasil Adugna Kendie; fasiladugna@gmail.com

Received 1 October 2020; Revised 4 February 2021; Accepted 26 February 2021; Published 4 March 2021

Academic Editor: Hassan Vatandoost

Copyright ( 92021 Fasil Adugna Kendie et al. This is an open access article distributed under the Creative Commons Attribution License, which permits unrestricted use, distribution, and reproduction in any medium, provided the original work is properly cited.

\begin{abstract}
Background. Malaria is one of the leading causes of mortality and morbidity in tropical and subtropical regions. The bulk of the global malaria burden is in sub-Saharan African countries, including Ethiopia. Malaria adversely affects the health of the peoples as well as the economic development of many developing countries including Ethiopia. Methods. This review article was reported according to PRISMA guidelines. Related published articles were searched from online public databases, such as PubMed, Google Scholar, and ScienceDirect. The search approach used to retrieve related articles were "prevalence," "malaria," "adults," and "Ethiopia." The quality of articles was assessed using Joana Brigg's Institute (JBI) critical appraisal checklist. The meta-analysis was computed using STATA version 14 . The pooled prevalence estimates with $95 \%$ confidence interval were analyzed using a randomeffect model, and the possible source of heterogeneity across studies was indicated through subgroup analysis, inverse of variance $\left(I^{2}\right)$, and time series analysis. The presence of publication bias was evaluated using funnel plots and Egger's regression test. Results. Out of 144 studies collected, only eight full-text articles were screened and included in the final quantitative meta-analysis. The pooled prevalence of malaria among adults in Ethiopia was 13.61\%. Subgroup analysis based on types of malaria cases showed that the prevalence of malaria among symptomatic and asymptomatic adults was $15.34 \%$ and $11.99 \%$, respectively. Similarly, regional subgroup analysis showed that the highest malaria prevalence was recorded in Southern Nations, Nationalities, and Peoples' Region (SNNPR) (16.17\%) followed by Oromia Regional State (13.11\%) and Amhara Regional State (12.41\%). Discussion and Conclusion. The current systematic review and meta-analysis showed that the pooled prevalence of malaria among adults was found to be greater than the general population and nearly equal to pregnant women. Therefore, the current prevention and control measures, which are related to both vectors and parasites, should be strengthened.
\end{abstract}

\section{Background}

Malaria is a protozoan disease caused by Plasmodium parasites [1, 2], which is one of the leading causes of mortality and morbidity in many developing countries $[3,4]$. An estimated 3.3 billion people are at risk of malaria worldwide [5], and it is a major health problem in tropical and subtropical regions [6]. World Health Organization estimates about 229 million new cases of malaria in 2019 in the world. Most of these malaria cases were in the WHO African region (94\%). Likewise, there were 409,000 malaria deaths estimated in the globe. Most of these deaths occurred in the WHO Africa region (94\%) [7]. Malaria is one of the major diseases of poor people in developing countries. The bulk of the global malaria burden is in sub-Saharan Africa, with the highest global cases and deaths. It is adversely affecting the health of the peoples as well as the economic development of many developing countries, particularly in sub-Saharan Africa [7-9].

Malaria causes serious complications, such as severe anaemia, cerebral malaria, acute renal failure, and hypoglycemia among infected individuals [10]. It is caused by five species of the genus Plasmodium [11]. Among these, four species including $P$. falciparum, $P$. vivax, $P$. ovale, and 
P. malariae are known to infect human beings in Ethiopia [12]. From the four Plasmodium species, P. falciparum is more severe in morbidity and mortality followed by $P$. vivax [13] with proportions of $60 \%$ and $40 \%$, respectively [14].

Malaria is transmitted by the biting of an infected female Anopheles mosquito, which serves as a vector of Plasmodium species [1, 15]. There are more than forty species of Anopheles mosquitoes in Ethiopia [16-18] of which Anopheles arabiensis, Anopheles funestus, Anopheles pharoensis, and Anopheles nili are the malaria vectors. A. arabiensis is the primary malaria vector, while $A$. funestus and $A$. pharoensis are considered as secondary vectors in Ethiopia [19, 20].

In Ethiopia, malaria is one of the main public health and economic problems. The distribution varies from place to place depending on climate, rainfall patterns, and altitude $[8,21]$. It is a major concern in the country, and it is one of the leading causes of morbidity and mortality. In Ethiopia, $75 \%$ of the landscape areas below $2000 \mathrm{~m}$ above the sea level are affected by malaria cases [22]. The major epidemics occur cyclically in every 5-8 years in Ethiopia, but focal epidemics are occurring every year [23]. Ethiopia has a population of above 100 million, of which approximately $68 \%$ of the population is at risk of the disease $[22,24]$. About 2.9 million cases of malaria and 4,782,000 related deaths have been reported annually, and the rate of morbidity and mortality dramatically increases during epidemics $[22,25]$.

Ethiopia is one of the countries that have implemented the revised strategies to control malaria. Among these, indoor residual spraying (IRS) and long-lasting insecticidal nets (LLINs) are the most important in malaria prevention and control strategy [26]. Additionally, introduction of rapid diagnostic tests at community level and adaptation of artemisinin-based combination therapies (ACTs) are also practiced in Ethiopia [27]. Despite the massive efforts to implement these strategies, malaria continues to cause significant morbidities and mortalities in the endemic foci of Ethiopia. On the other hand, the developments of insecticide resistance on IRS and LLINS in different parts of the country have its own impact to control the main malaria vectors. In Ethiopia, the development of resistance in different insecticide groups on A. arabiensis was reported by Yewhalaw and his colleague [26], Abate and Hadis [28], Massebo and Lindtjørn [29], and others.

Malaria causes much damage to the health and socioeconomic development of the country [22]. The disease is more severe in children and pregnant women in the country [7]. Although several malaria prevalence studies in different parts of Ethiopia have focused on all age groups of individuals, only a few studies have indicated that adulthood malaria is still one of the major public health problems in the country. To date, however, the national estimate of adulthood malaria in the country is not known.

Systematic review and meta-analysis generate concrete evidence in which the evidence may help policymakers and program managers to design appropriate intervention to control and minimize the negative consequences of adulthood malaria. Furthermore, there is no published systematic review and meta-analysis that generated the pooled estimate of the prevalence of malaria at adulthood stage in Ethiopia.
Therefore, the aim of this study was to determine the pooled prevalence of adulthood malaria in Ethiopia from studies conducted between 2010 and May 2020.

\section{Methods}

2.1. Search Strategy. This review article was reported according to the Preferred Reporting Items for Systematic Reviews and Meta-Analyses (PRISMA) guidelines. Related published articles were searched from online public databases such as PubMed, Google Scholar, and ScienceDirect, which reported the prevalence of malaria among adults in Ethiopia from 2010 to May 2020.

The search terms were used in agreement with the Medical Subject Heading (MeSH) using the arrangement of key words which were used to select related research articles. The search terms were used separately and in combination using Boolean operators like "OR" or "AND." The search approach used to retrieve related articles was as follows: ((Prevalence) OR prevalence [MeSH Terms]) AND malaria) OR malaria [MeSH Terms]) (AND adults) OR adults [MeSH Terms]) AND Ethiopia) OR Ethiopia [MeSH Terms]). The repeated articles were excluded in the systematic and metaanalysis review. The software EndNote version X5 (Thomson Reuters, New York, NY) was used to arrange references and remove repeated references.

\subsection{Eligibility Criteria}

2.2.1. Inclusion Criteria. Studies published in the peerreviewed journals, which reports the prevalence of malaria among adults, were included. All studies were original research articles published in English and contained the basic information concerning sample size, diagnostic methods, prevalence, and status of malaria infection among adults in different parts of Ethiopia. Moreover, studies that have been carried out in health institution and community-based settings, symptomatic or asymptomatic individuals, and coinfections (malaria and HIV/AIDS) as far as they reported malaria among adults were included this review.

2.2.2. Exclusion Criteria. Studies conducted among all age groups, pregnant women, children, unknown malarial detection methods, unknown sample size, and lack of clear figure about infected cases were excluded from this review.

2.3. Search Methods and Quality Assessment. Two authors (FA and TH) individually conducted a search in PubMed, Google Scholar, and ScienceDirect using the keywords. The searched articles were screened by the title and abstract to transfer the articles in the full-text review. The quality of articles was assessed using Joana Brigg's Institute (JBI) critical appraisal checklist for simple prevalence [30]. The two authors (FA and TH) independently assessed the quality of journals included in this review. The differences in the inclusion and quality of individual articles between the two authors were resolved by discussion with the third author (EN). 


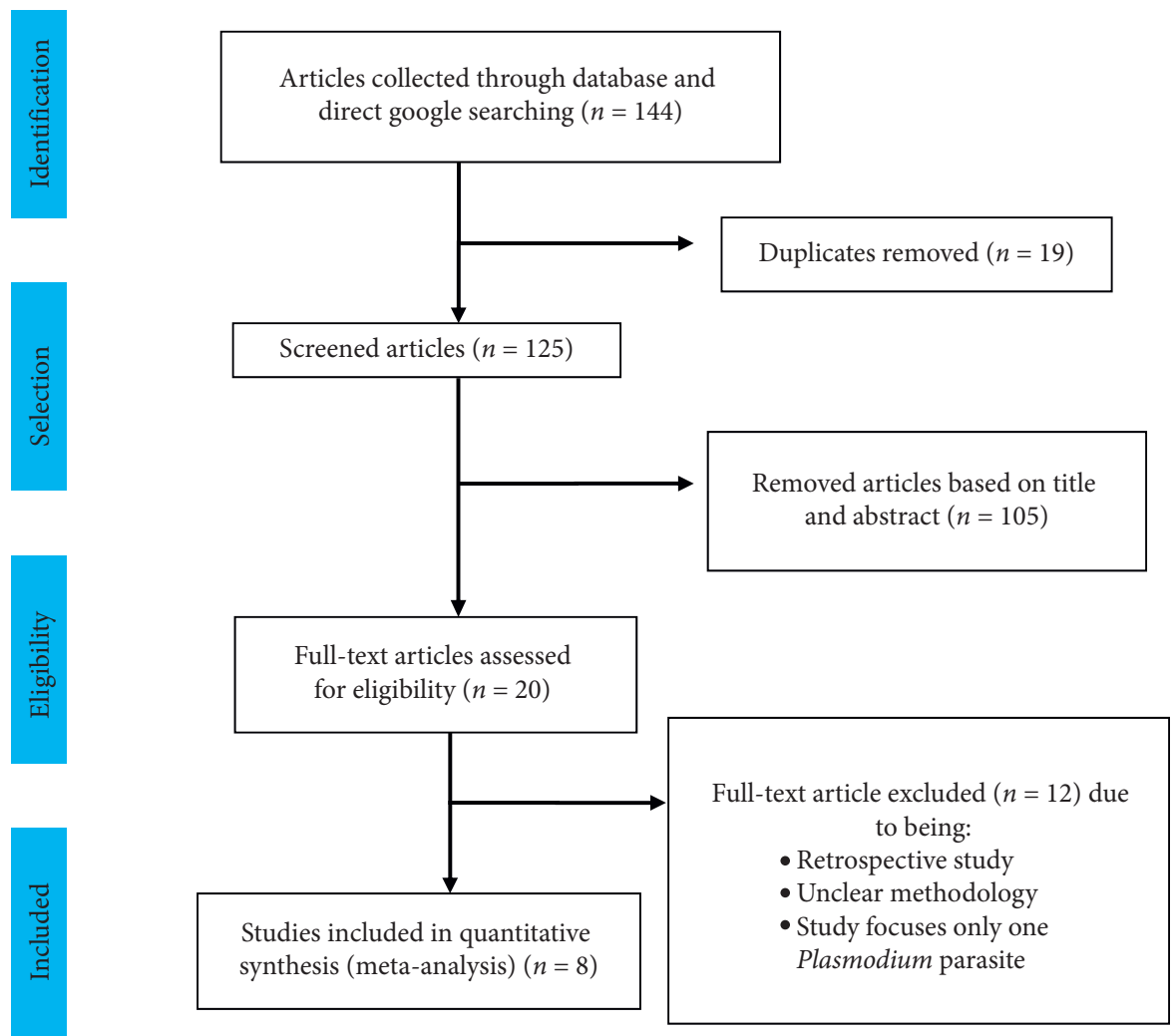

FIgURE 1: PRISMA flow diagram showing the selection process of eligible studies for this review, 2020.

2.4. Data Extraction. Data extraction protocol was developed by two authors (FA and TA) and evaluated by EN and MW. This extraction protocol consists of name of the first author, year of publication, study area (region), study group, study design, sample size, prevalence of malaria, prevalence of $P$. falciparum, the prevalence of $P$. vivax, prevalence of mixed infection, and type of the diagnostic method used.

2.5. Data Analysis. Eligible primary studies were extracted, entered into Microsoft Excel, and then exported to STATA version 14. Forest plots were used to estimate the pooled effect size and effect of each study with their confidence interval (CI) and to provide a visual image of the data. The degree of heterogeneity between the included studies was evaluated by the inverse of variance $\left(I^{2}\right)$ [31]. $I^{2}$ values of $25 \%, 50 \%$, and $75 \%$ were assumed to represent low, medium, and high heterogeneity, respectively. Due to the observed high heterogeneity across studies, we used a random effect model to assess pooled estimate. The analysis between the subgroups was carried out regarding the type of cases. Small study effect and publication bias across studies were evaluated by the funnel plot symmetry subjectively and Egger's regression test objectively.

\section{Results}

3.1. Selection and Characterization of Included Studies. This systematic review contains published articles on the prevalence of malaria among adults. A total of 144 articles were retrieved from online databases using manual searching. From these studies, 19 articles were excluded due to duplication records. From the remaining 125 articles, 105 of them were excluded by evaluation of their title and abstract. The remaining 20 articles were eligible for full-text assessment. From the 20 eligible articles, 12 studies were excluded with specific exclusion criteria such as being retrospective study, studies with unclear methodology, and studies focusing only one Plasmodium parasite. Finally, only eight full-text articles were screened for eligibility and included in the final quantitative meta-analysis (Figure 1).

Eight original full-text articles were included in this systematic review and meta-analysis. A total of 7895 study participants were tested for malaria infection from the eligible articles for this review. Among these, 775 were positive for malaria infection (377, 368, and 30 for $P$. falciparum, $P$. vivax, and mixed infections, respectively) (Supplementary file 2). Seven of the eight articles were used cross-sectional design, and the remaining one study used longitudinal research design. The sample sizes included in the eligible studies were ranged from 385 [32] to 3638 [33] populations. All studies were used for microscopic examination except for one study, which used RDT. All studies included in this review were carried out in three regions: $37.5 \%$ from Amhara Regional State, 37.5\% from Oromia Regional State, and the remaining 25\% from Southern Nations and Nationalities Peoples' Regional State (SNNPR). Unfortunately, there was no study found from other Regional States and city administrations such as Tigray, Afar, Somali, Gambella, Harari, Benshangul Gumuz, Addis Ababa, and Dire Dawa city administrations. 


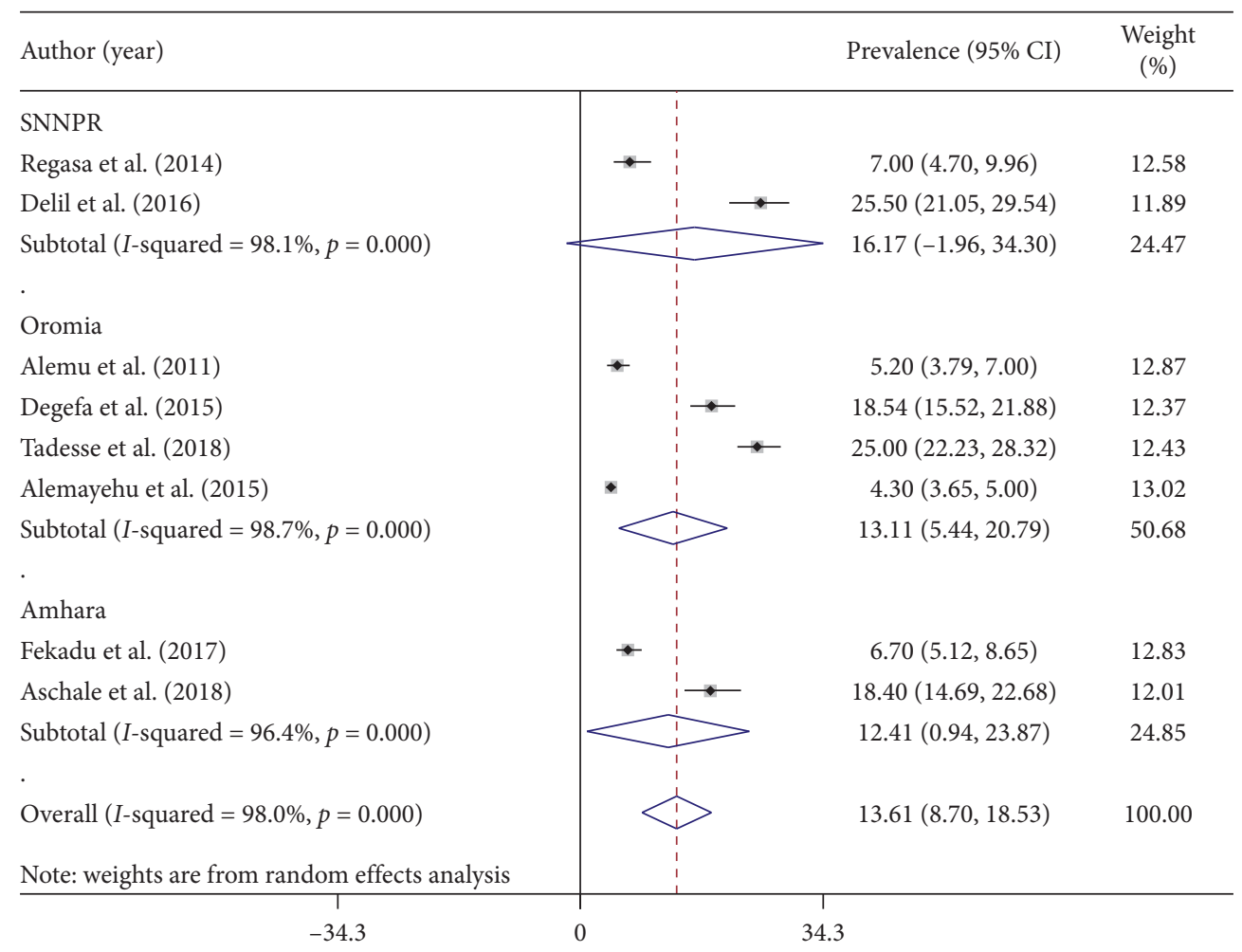

FIgURE 2: Forest plot showing the combined malaria pooled prevalence estimate among adults by regions of study in Ethiopia, 2020.

3.2. Quality Assessment. All studies were evaluated with nine criteria of the JBI quality assessment tool for the prevalence studies included in this review. The result of the analysis showed that the included studies had low risk of bias because its total score is $72 \%$, which is greater than $50 \%$ (Supplementary file 1).

3.3. Prevalence of Malaria among Adult Stages. Eight articles were selected for this systematic review and meta-analysis, which were used to estimate the pooled prevalence of malaria among adults. The minimum prevalence of malaria was $4.3 \%$ observed in Oromia Regional State of different hospitals and health center [33], while the maximum malaria parasites was $25.5 \%$ observed in SNNPR of Hadiya Zone Health Centers [34]. The pooled prevalence of malaria among adults in Ethiopia was 13.61\% (95\% CI: 8.70-18.53) (Figure 2$)$. High heterogeneity $\left(I^{2}=98 \%\right.$ and $p$ value $\left.\leq 0.001\right)$ across studies was observed in the analysis.

3.4. Subgroup Analysis Based on Symptoms and Regions of Study. Subgroup analysis based on types of malaria cases showed that the prevalence of malaria among symptomatic and asymptomatic adults was $15.34 \%$ (95\% CI: 4.66-26.01) and $11.99 \%$ (95\% CI: 5.92-18.07), respectively (Figure 3). Similarly, regional subgroup analysis showed that the highest malaria prevalence was recorded in SNNPR (16.17\%) (95\% CI: $-1.96-34.30)$ followed by Oromia Regional State (13.11\%) (95\% CI: 5.44-20.79), and the least was documented in Amhara Regional State (12.41\%) (95\% CI: 0.94-23.87) (Figure 2).
3.5. Prevalence of Malaria Parasite Species among Adults. The prevalence of Plasmodium parasite species was compared between studies among Ethiopian adults. All of the studies [8] included in this review reported the prevalence of $P$. vivax and $P$. falciparum infection, while the prevalence of mixed infection was reported from seven studies. The overall pooled prevalence of Plasmodium species was as follows: P. falciparum $(6.48 \%)$ (95\% CI: 4.13-8.82), P. vivax (5.74\%) (95\% CI: 3.53-7.95), and mixed infection (0.47\%) (95\% CI: 0.09-0.85) (Figure 4).

3.6. Publication Bias across Studies. The presence of publication bias was evaluated subjectively using funnel plots symmetry and objectively using Egger's regression test. The symmetrical distribution of the funnel plot indicated the absence of publication bias across studies (Figure 5 ). The result of Egger's regression test $(p$ value $=0.262$ ) indicated there was no evidence of publication bias across studies.

3.7. Time Series Analysis. The trend in malaria prevalence among included studies did not uniformly change within the ten years (2011-2018). From 2011 to 2014, the prevalence was characterized by a slight change in either direction (right and left). On the other hand, from 2016 to 2018, the prevalence showed an abrupt decrement and increment compared to the previous years (Figure 6).

\section{Discussion}

Despite the declining of malaria in Ethiopia, the disease is still a major public health concern, and it is one of the 


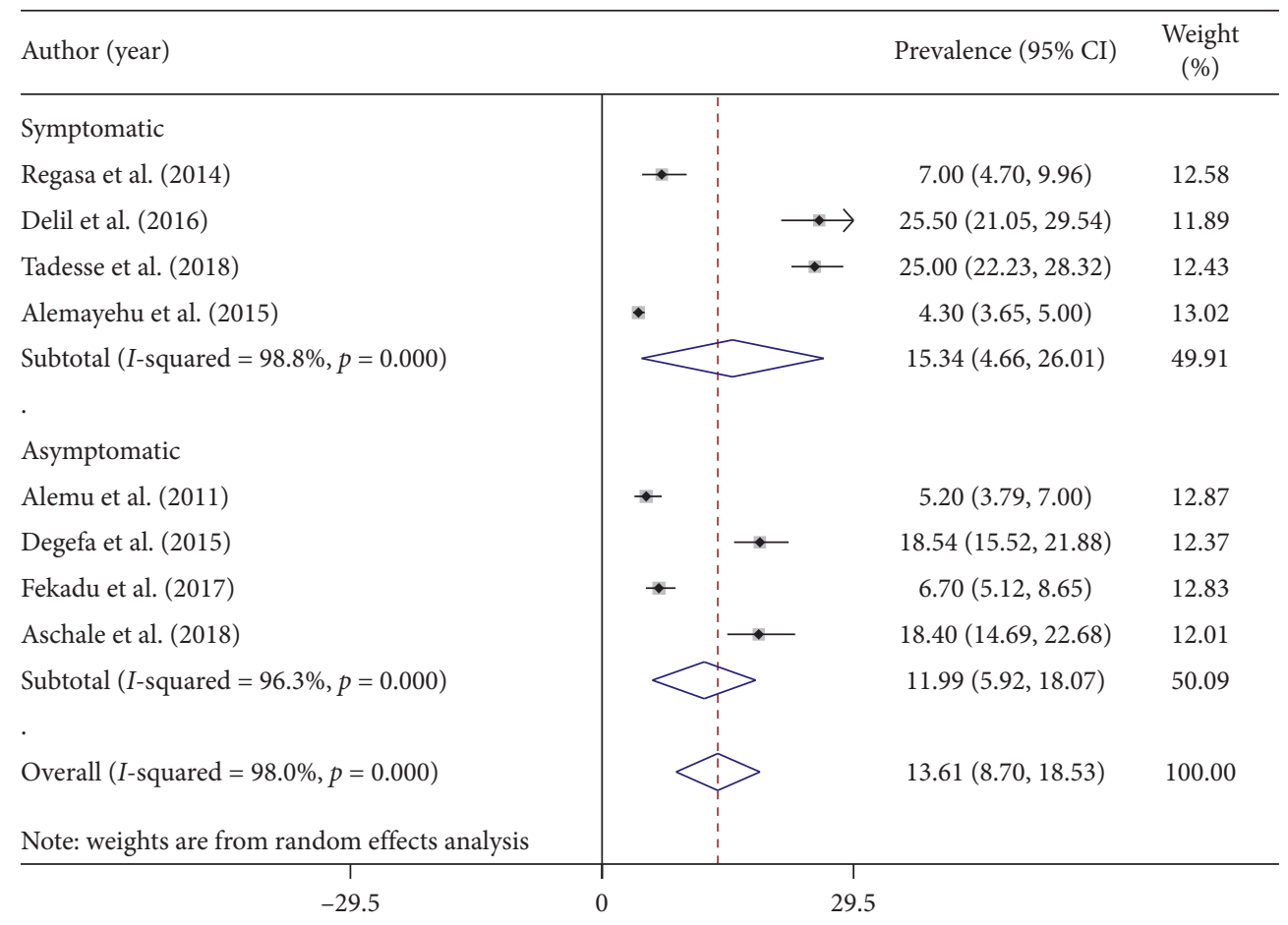

FIGURE 3: Forest plot showing the combined malaria pooled prevalence estimate among adults by types of study participants in Ethiopia, 2020.

leading causes of morbidity and mortality among adult populations $[34,35]$, especially the productive age groups (15-45 years) were more exposed to Plasmodium infections in some areas [36]. This might be associated with respondents in these age groups who are likely to have lower malaria immunity than those in higher age groups. Moreover, higher exposure to outdoor activities of adults before bed time exposes more to the infection [37].

The current systematic review and meta-analysis was conducted using eight full-text articles to determine the pooled prevalence of malaria among adults in Ethiopia. Malaria causes serious complications in human, such as severe anaemia, acute renal failure, hypoglycemia [10], loss of productivity, school absenteeism, and other complications [38]. Therefore, the accurate malaria prevalence information is vital for the proper diagnosis, treatment, prevention, and policy preparation [39].

This systematic review and meta-analysis study showed that the pooled prevalence of malaria among adults in Ethiopia was $13.61 \%$. This is much greater than the 2015 malaria indicator survey among the general population of the country, which results in $1.2 \%$ and $0.5 \%$ malaria prevalence by RDT and microscopy tests, respectively [40]. This study is nearly similar to systematic review and metaanalysis conducted among pregnant women in Ethiopia with the pooled prevalence of malaria $12.72 \%$ [41]. On the other hand, the result of this study was much lower than the previous research studies reported in Ethiopia among all age groups [39, 42].

The comparative decrement of malaria prevalence in the current study might be related to life style change, geographical area, economic status, the type of malaria diagnosis methods used, and the time at which the study conducted [33]. In addition to this, the malaria elimination and control program, such as proper use of long-lasting insecticide-treated nets (LLIN), insecticide residual spraying (IRS), introduction of rapid diagnostic tests at community level, and adaptation of artemisinin-based combination therapies (ACTs), might have led to a reduction in the burden of malaria in Ethiopia [27].

Subgroup analysis based on types of malaria cases showed that the prevalence of malaria among symptomatic and asymptomatic adults were $15.45 \%$ and $11.98 \%$, respectively. The difference in the prevalence of malaria between the two groups is associated with parasite load; there might be higher parasite load among symptomatic cases than asymptomatic cases [41]. Similarly, regional subgroup analysis showed that the highest malaria prevalence was recorded in SNNPR (16.17\%) followed by Oromia Regional State $(13.25 \%)$ and the least was documented in Amhara Regional State $(12.41 \%)$. This heterogeneity could be observed due to differences in methodology, sample size and sampling technique, and study participant characteristics. In addition, these variations could be explained by the existing differences in the environmental condition, rainfall, climate condition, residents' lifestyles, existence of swampy and irrigation areas, and the extent to which prevention and control measures are applied [41]. Moreover, the most likely reason for this variation is that some of the studies were obtained from the high malaria-endemic areas of the country, while others were obtained from medium and low malaria-risk areas. Furthermore, studies were conducted in 


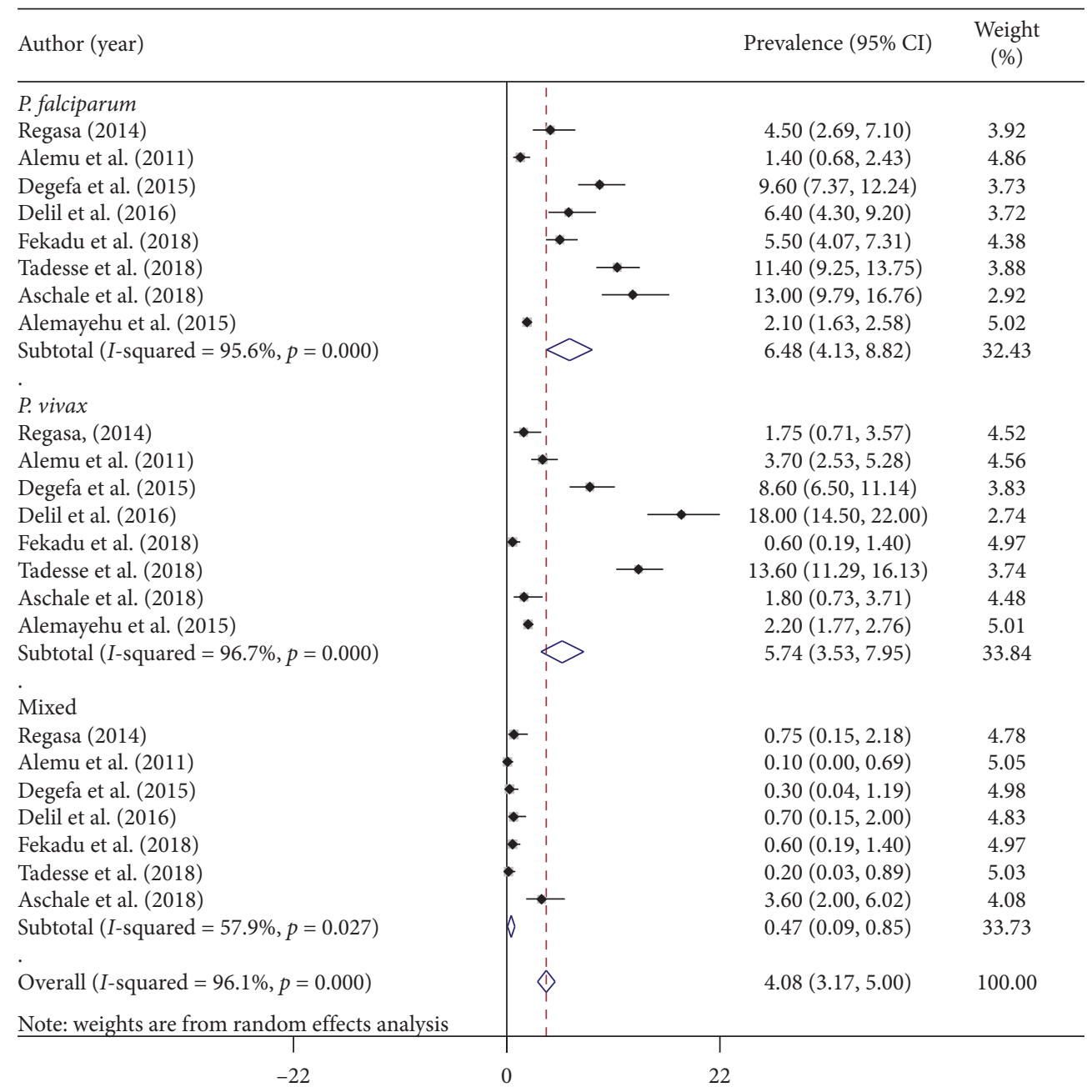

FIGURE 4: Forest plot showing malaria pooled prevalence estimate among adults by Plasmodium parasites in Ethiopia, 2020.

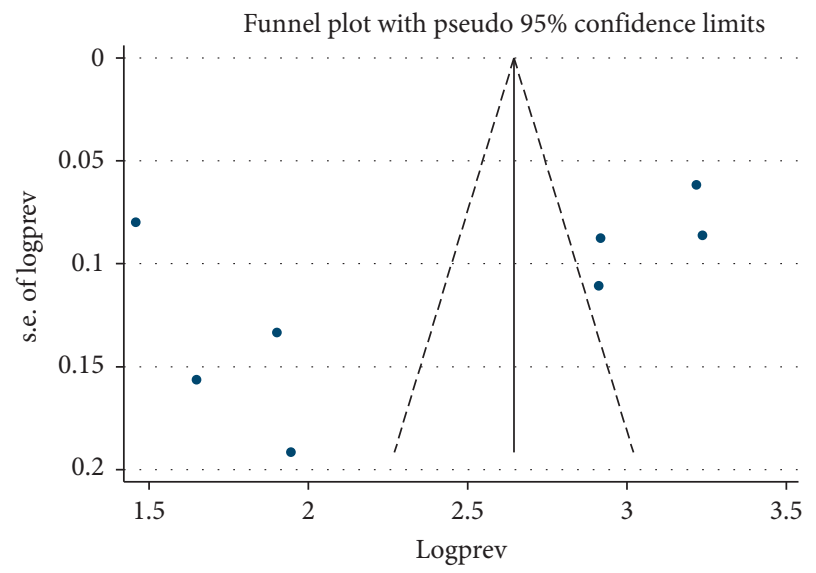

FIGURE 5: Funnel plots indicate absence of publication bias across studies included in this review in Ethiopia, 2020.

different malaria transmission seasons, which means some studies were conducted during the high transmission periods while others were conducted during the least transmission seasons [33] which could be a significant contributing factor for the high variations [39]. On the other

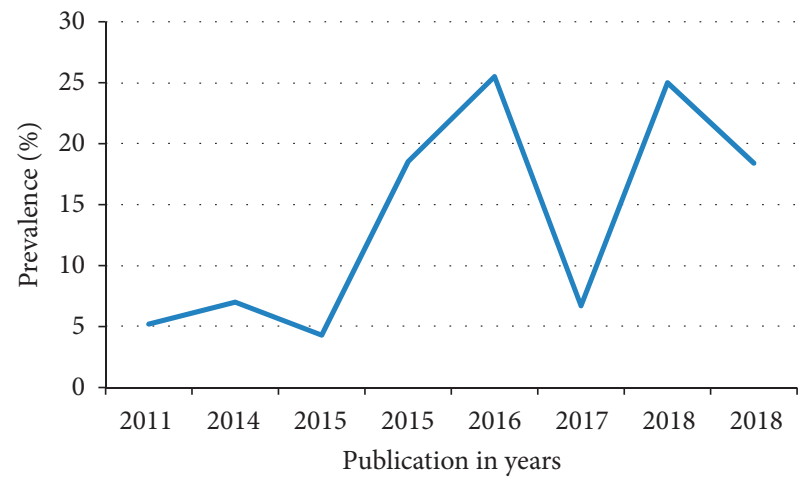

FIgURE 6: Time trend analysis of adult malaria prevalence in Ethiopia from 2011 to 2018.

hand, the variations could be due to the presence of different topographic platforms that control the multiplication rate and diversity of malaria vectors [43].

The trend of malaria prevalence among studies included in this review did not uniformly change within the ten years (2010-2019) (Figure 6). However, this discrepancy in prevalence estimates over time could be due to the fact that 
malaria infection in Ethiopia is highly variable and unstable and the occurrence of epidemics over several locations (agroecological regions) of the country [39].

The overall pooled prevalence of $P$. falciparum (6.48\%) and $P$. vivax $(5.74 \%)$ parasites was found in proportions of $48.6 \%$ and $47.5 \%$, respectively. The result of this study showed that $P$. falciparum and $P$. vivax have nearly equal contributions to the disease, malaria. This is similar to a study conducted in Batu town, Oromia Regional State [44]. On the other hand, the results contradicted to the previous reports in which $P$. falciparum proportion is greater than those of $P$. vivax in Ethiopia [23, 40, 42, 45]. In addition, it also contradicted the study that showed the prevalence of $P$. vivax is higher than $P$. falciparum [46-48]. Almost equal proportions of $P$. falciparum and $P$. vivax infections in our study indicate a shift in the trend of species composition in Ethiopia.

4.1. Limitations. Malaria is an important public health problem among adult individuals in Ethiopia. This study summarized the prevalence of malaria among adult in Ethiopia that can be an input for policymakers. Despite this, the present study has few limitations. First, a small number of articles included in this systematic review and metaanalysis could affect the pooled prevalence estimate. Second, almost all of the included studies were cross-sectional studies, and due to such reasons, the outcome variable could be affected by other confounding variables. Third, half of the included articles were obtained from the Oromia Regional State, whereas the other half was found to be in Amhara Regional State and SNNPR. This unequal distribution of articles throughout the country may affect the outcomes of this study. Fourth, no study obtained from Afar, Benishangul Gumuz, Gambella, Harari, and Tigray regions. These aforementioned limitations might affect the results reported in this review regarding the overall prevalence of malaria among adults in Ethiopia.

\section{Conclusions}

The current systematic review and meta-analysis showed that the pooled prevalence of malaria among adults was found to be greater than the general population and is nearly equal to pregnant women. Therefore, the existing prevention and control measures such as health education, usage of LLIN and application of IRS for controlling the vector, early diagnosis and treatment, and adaptation of artemisininbased therapies should be adopted. In addition to this, Ethiopian public health institution and other responsible bodies should focus on the substantial reduction of mosquito-breading sites through community participation. Moreover, monitoring and mapping the distribution of insecticide resistance is appropriate in order to develop new vector control strategies.

\section{Data Availability}

The data used and analyzed during the current study are available within the manuscript and supplementary materials.

\section{Conflicts of Interest}

The authors declare that they have no conflicts of interest.

\section{Authors' Contributions}

FA proposed this systematic and meta-analysis project and collected the related articles using different online databases and sources. FA and TH organized the data in computer, did the analysis, interpretation, and wrote the manuscript. EN and MW revised the manuscript for scientific content and did the language check. All authors read and approved the final manuscript.

\section{Acknowledgments}

The authors would like to thank all online public databases that contributed to the data used in this study. Additionally, the authors would like to thank the College of Science, Bahir Dar University, which supplied Internet access and laptop computers.

\section{Supplementary Materials}

Table S1: the nine criteria of the JBI quality assessment tool used to evaluate this prevalence review study. Table S2: the characteristics of 8 eligible studies included in this metaanalysis. (Supplementary Materials)

\section{References}

[1] F. E. Cox, "History of the discovery of the malaria parasites and their vectors," Parasite Vectors, vol. 3, no. 1, pp. 1-9, 2010.

[2] R. W. Snow, C. A. Guerra, A. M. Noor, H. Y. Myint, and S. I. Hay, "The global distribution of clinical episodes of Plasmodium falciparum malaria," Nature, vol. 434, no. 7030, pp. 214-217, 2005.

[3] WHO, World Malaria Report, Britain and Bill Gates Fight Malaria, World Health Organization, Geneva, Switzerland, 2015, https://apps.who.int/iris/bitstream/handle.

[4] WHO, World Malaria Report, World Health Organization, Geneva, Switzerland, 2017, https://www.mmv.org/newsroom/ publications/world-malaria-report-2017.

[5] J. I. Nankabirwa, A. Yeka, E. Arinaitwe et al., "Estimating malaria parasite prevalence from community surveys in Uganda: a comparison of microscopy, rapid diagnostic tests and polymerase chain reaction," Malar Journal, vol. 14, no. 1, p. 528, 2015.

[6] K. Raghavendra, T. K. Barik, B. P. N. Reddy, P. Sharma, and A. P. Dash, "Malaria vector control: from past to future," Parasitology Research, vol. 108, no. 4, pp. 757-779, 2011.

[7] WHO, World Malaria Report, World Health Organization, Geneva, Switzerland, 2020, https://www.who.int/news-room/ fact-sheets/detail/malaria.

[8] J. Barofsky, C. Chase, T. Anekwe, and F. Farzadfar, "The economic effects of malaria eradication: evidence from an intervention in Uganda," Program on the Global Demography of Aging Working Paper, vol. 2011, no. 70, 2011.

[9] M. Castillo-Riquelme, D. McIntyre, and K. Barnes, "Household burden of malaria in South Africa and Mozambique: is there a catastrophic impact?" Tropical Medicine and International Health, vol. 13, no. 1, pp. 108-122, 2008. 
[10] J. K. Choge, G. M. Ng’Wena, W. Akhwale et al., "Symptomatic malaria diagnosis overestimate malaria prevalence, but underestimate anaemia burdens in children: results of a follow up study in Kenya," BMC Public Health, vol. 14, no. 1, p. 332, 2014.

[11] N. Byrne, "Urban malaria risk in sub-Saharan Africa: where is the evidence?" Travel Medicine and Infectious Disease, vol. 5, no. 2, pp. 135-137, 2007.

[12] A. S. Bedane, T. K. Tanto, and T. F. Asena, "Malaria distribution in Kucha district of Gamo Gofa Zone, Ethiopia: a time series approach," American Journal of Theoretical and Applied Statistics, vol. 5, no. 2, pp. 70-79, 2016.

[13] S. I. Hay, C. A. Guerra, A. J. Tatem, A. M. Noor, and R. W. Snow, "The global distribution and population at risk of malaria: past, present, and future," The Lancet Infectious Diseases, vol. 4, no. 6, pp. 327-336, 2004.

[14] K. Tsige, B. Ketema, B. Tarekegn, and P. Beyene, "Chloroquine-resistant Plasmodium vivax malaria in Serbo town, Jimma zone, south-west Ethiopia," Malar Journal, vol. 8, no. 1, p. 177, 2009.

[15] WHO, World Malaria Report, https://www.mmv.org/ newsroom/publications/world-malaria-report-2018, World Health Organization, Geneva, Switzerland, 2018, https:// www.mmv.org/newsroom/publications/world-malariareport-2018.

[16] R. H. Hunt, M. Coetzee, and M. Fettene, "The Anopheles gambiae complex: a new species from Ethiopia," Transactions of the Royal Society of Tropical Medicine and Hygiene, vol. 92, no. 2, pp. 231-235, 1998.

[17] FMoH, Five-Year National Operational Plan for Malaria Prevention and Control in Ethiopia 2016-2020, FMoH, Addis Ababa, Ethiopia, 2016.

[18] D. Kyalo, P. Amratia, C. W. Mundia, C. M. Mbogo, M. Coetzee, and R. W. Snow, "A geo-coded inventory of anophelines in the Afrotropical Region south of the Sahara: 1898-2016," Wellcome Open Research, vol. 2, 2017.

[19] NSPE, National Strategic Plan for Malaria Prevention Control and Elimination in Ethiopia, NSPE, Alexandria, VA, USA, 2010, https://extranet.who.int/countryplanningcycles/sites/ default/files/country_docs/Ethiopia/ethiopia_malaria_ national_strategic_plan_2011-2015.

[20] USAID, President's Malaria Initiative Ethiopia, Malaria Operational Plan, https://reliefweb.int/report/ethiopia/ president-s-malaria-initiative-ethiopia-malaria-operationalplan-fy-2017, USAID, Washington, DC, USA, 2017, https:// reliefweb.int/report/ethiopia/president-s-malaria-initiativeethiopia-malaria-operational-plan-fy- 2017.

[21] L. Yared, T. Ayalew, B. Tefera, and K. Tushune, "Knowledge, attitude and practice about malaria transmission and its preventive measures among households in urban areas of Assosa Zone, Western Ethiopia," Ethiopian Journal of Health Devevelopment, vol. 22, no. 2, pp. 157-165, 2007.

[22] T. Girum, T. Shumbej, and S. Misgun, "Burden of malaria in Ethiopia, 2000-2016: findings from the Global Health Estimates 2016," Tropical Diseases, Travel Medicine and Vaccines, vol. 5, no. 1, p. 11, 2019.

[23] K. Tsige, G. Kefelegn, and B. Ketema, "Therapeutic efficacy of chloroquine for treatment of Plasmodium vivax malaria cases in Halaba district, South Ethiopia," Parasites and Vectors, vol. 4, no. 1, p. 46, 2011.

[24] WHO, World Malaria Report, World Health Organization, Geneva, Switzerland, 2016, https://www.mmv.org/newsroom/ publications/world-malaria-report-2016.
[25] N. Alemayehu, H. Gadissa, G. Dawit et al., "Can training health extension workers in the integrated pharmaceutical logistics system (IPLS) be effective, affordable, and opportunistic," Ethiopian Medical Journal, vol. 52, no. suppl 3, pp. 11-12, 2014.

[26] D. Yewhalaw and E. J. Kweka, "Insecticide resistance in East Africa-history, distribution and drawbacks on malaria vectors and disease control," in Insecticides Resistance, pp. 189-215, INTECH Science, Technology and Medicine Open Access, London, UK, 2016.

[27] O. Kenea, M. Balkew, H. Tekie et al., "Impact of combining indoor residual spraying and long-lasting insecticidal nets on Anopheles arabiensis in Ethiopia: results from a cluster randomized controlled trial," Malar Journal, vol. 18, no. 1, p. 182, 2019.

[28] A. Abate and M. Hadis, "Susceptibility of Anopheles gambiae s.l. to DDT, malathion, permethrin and deltamethrin in Ethiopia," Tropical Medicine \& International Health, vol. 16, no. 4, pp. 486-491, 2011.

[29] F. Massebo and B. Lindtjørn, "The effect of screening doors and windows on indoor density of Anopheles arabiensis in south-west Ethiopia: a randomized trial," Malaria Journal, vol. 12, no. 1, pp. 1-6, 2013.

[30] Z. Munn, S. Moola, K. Lisy, D. Riitano, and C. Tufanaru, "Methodological guidance for systematic reviews of observational epidemiological studies reporting prevalence and cumulative incidence data," International Journal of EvidenceBased Healthcare, vol. 13, no. 3, pp. 147-153, 2015.

[31] J. P. T. Higgins and S. G. Thompson, "Quantifying heterogeneity in a meta-analysis," Statistics in Medicine, vol. 21, no. 11, pp. 1539-1558, 2002.

[32] Y. Aschale, A. Mengist, A. Bitew, B. Kassie, and A. Talie, "Prevalence of malaria and associated risk factors among asymptomatic migrant laborers in West Armachiho District, Northwest Ethiopia," Research and Reports in Tropical Medicine, vol. 9, pp. 95-101, 2018.

[33] G. Alemayehu, Z. Melaku, T. Abreha et al., "Burden of malaria among adult patients attending general medical outpatient department and HIV care and treatment clinics in Oromia, Ethiopia: a comparative cross-sectional study," Malar Journal, vol. 14, no. 1, p. 501, 2015.

[34] R. K. Delil, T. K. Dileba, Y. A. Habtu, T. F. Gone, and T. J. Leta, "Magnitude of malaria and factors among febrile cases in low transmission areas of Hadiya zone, Ethiopia: a facility based cross sectional study," PLoS One, vol. 11, no. 5, 2016.

[35] E. M. Belete and A. B. Roro, "Malaria prevalence and its associated risk factors among patients attending Chichu and Wonago Health Centres, South Ethiopia," Journal of Research in Health Sciences, vol. 16, no. 4, pp. 185-189, 2016.

[36] T. Frew, A. W. Fogarty, and W. Deressa, "Prevalence and associated risk factors of malaria among adults in East Shewa Zone of Oromia Regional State, Ethiopia: a cross-sectional study," BMC Public Health, vol. 18, no. 1, p. 25, 2018.

[37] F. Mesafint, M. K. Yenit, and A. M. Lakew, "The prevalence of asymptomatic malaria parasitemia and associated factors among adults in Dembia district, northwest Ethiopia, 2017," Archives of Public Health, vol. 76, no. 1, p. 74, 2018.

[38] A. Alemu, D. Muluye, M. Mihret, M. Adugna, and M. Gebeyaw, "Ten year trend analysis of malaria prevalence in kola diba, north Gondar, northwest Ethiopia," Parasit Vectors, vol. 5, no. 1, p. 173, 2012.

[39] T. Deress and M. Girma, "Plasmodium falciparum and Plasmodium vivax prevalence in Ethiopia: a systematic review 
and meta-analysis," Malaria Research and Treatment, vol. 2019, Article ID 7065064, 12 pages, 2019.

[40] FMoH, Ethiopia National Malaria Indicator Survey, Ethiopian Public Health Institute, Addis Ababa, Ethiopia, 2016.

[41] Y. Tegegne, D. Asmelash, S. Ambachew, S. Eshetie, A. Addisu, and A. Jejaw Zeleke, "The prevalence of malaria among pregnant women in Ethiopia: a systematic review and metaanalysis," Journal of Parasitology Research, vol. 2019, Article ID 8396091, 9 pages, 2019.

[42] A. Assefa, A. A. Ahmed, W. Deressa et al., "Multiplex serology demonstrate cumulative prevalence and spatial distribution of malaria in Ethiopia," Malar Journal, vol. 18, no. 1, p. 246, 2019.

[43] Y. E.-S. Himeidan and E. Kweka, "Malaria in East African highlands during the past 30 years: impact of environmental changes," Frontiers in Physiology, vol. 3, p. 315, 2012.

[44] J. Hassen and H. Dinka, "Retrospective analysis of urban malaria cases due to Plasmodium falciparum and Plasmodium vivax: the case of Batu town, Oromia, Ethiopia," Heliyon, vol. 6, no. 3, Article ID e03616, 2020.

[45] W. Deressa, S. Chibsa, and D. Olana, "Treatment seeking of malaria patients in east shewa zone of Oromia, Ethiopia," Ethiopian Journal of Health Devevelopment, vol. 17, no. 1, pp. 9-15, 2003.

[46] A. Alemu, G. Abebe, W. Tsegaye, and L. Golassa, "Climatic variables and malaria transmission dynamics in Jimma town, South West Ethiopia," Parasit Vectors, vol. 4, no. 1, p. 30, 2011.

[47] S. Tesfaye, Y. Belyhun, T. Teklu, T. Mengesha, and B. Petros, "Malaria prevalence pattern observed in the highland fringe of Butajira, Southern Ethiopia: a longitudinal study from parasitological and entomological survey," Malar Journal, vol. 10, no. 1, p. 153,2011

[48] G. Tefera, "Prevalence of malaria and associated factors among patients attending at Hallaba Health Center, Southern Ethiopia," Immunology and Infectious Disease, vol. 2, no. 3, pp. 25-29, 2014. 